\title{
Diversity among isolates of Actinobacillus equuli and related organisms as revealed by ribotyping
}

\author{
PJ BLACKALL ${ }^{\mathrm{a}}$, JP CHRISTENSEN ${ }^{\mathrm{b}}$ and M BISGAARD ${ }^{\mathrm{b}}$
}

Objective The objective of this work was to examine the diversity within Australian isolates of Actinobacillus equuli and related organisms by the genotypic method of ribotyping.

Design Ribotyping, performed using the enzyme Haelll, was used to examine the diversity in 12 field isolates of $A$ equuli (five being capable of fermenting L-arabinose), one field isolate of Pasteurella caballi and two unclassifiable field isolates. Isolates were obtained from Australian horses, except for three isolates of $A$ equuli (one L-arabinose positive and two L-arabinose negative) which were obtained from horses and a pig in Africa. In addition, the type strains for $A$ equuli and $P$ caballi and a reference strain for Bisgaard Taxon 9 were included in the study.

Results The ribotype patterns were analysed by computerised cluster analysis, yielding five clusters (A to $E$ ). All five of the $\mathrm{L}$-arabinose positive $A$ equuli were assigned to cluster $\mathrm{A}$, with all the other seven $A$ equuli isolates (all L-arabinose negative) and the type strain being assigned to cluster $B$. One of the two unclassified isolates formed cluster $C$ along with the reference strain for Bisgaard Taxon 9. The remaining unclassified isolate formed cluster D. Cluster E consisted of the field isolate and reference strain of $P$ caballi.

Conclusion The results of this study indicate that $A$ equuli is a diverse species, with $\mathrm{L}$-arabinose positive isolates of $A$ equuli being quite distinct from typical $L$-arabinose negative isolates. Ribotyping appears to be a useful tool in confirming the identity of $A$ equuli-like organisms from horses.

Aust Vet J 1998;76:423-425

Key words: Actinobacillus equuli, ribotyping, L-arabinose.

$\angle$ ctinobacillus equuli is an opportunistic pathogen of horses and pigs, found in association with various clinical conditions, especially in young animals. The most usual disease syndrome associated with the organism in foals is an acute, highly fatal septicaemia of newborn foals (sleepy foal disease) that may become chronic (joint ill) with lesions of purulent nephritis and arthritis. ${ }^{1-3}$ There is evidence of a degree of diversity within the species, with some isolates of $A$ equuli being L-arabinose positive. ${ }^{1}$ In other species of the family Pasteurellaceae, the ability to ferment arabinose has been shown to have taxonomic significance. As an example, the species Haemophilus avium, which included both L-arabinose positive and negative strains, has been split on the basis of DNA-DNA hybridisation data into three taxa (Pasteurella avium, Pasteurella volantium and Pasteurella species A), with all L-arabinose positive strains being allocated to Pasteurella species A. ${ }^{4}$ Production of acid from L-arabinose has also been shown to be of taxo-

\footnotetext{
a Department of Primary Industries, Animal Research Institute, Yeerongpilly Queensland 4105

${ }^{b}$ Department of Veterinary Microbiology, Royal Veterinary and Agricultural University, Copenhagen, Denmark
}

nomic significance within the Pasteurella haemolytica complex. ${ }^{5}$

Ribotyping has been proposed as a useful tool in examining the diversity present in species within the family Pasteurellaceae. . $^{-10}$ There have been no previous reports of the use of ribotyping to examine isolates of $A$ equuli. The current study describes the use of ribotyping on a collection of L-arabinose positive and L-arabinose negative $A$ equuli isolates. The majority of the organisms used were from a recent study of Australian isolates which also included related organisms, specifically an Australian isolate of Pasteurella caballi as well as two Australian isolates that cannot be assigned to any currently recognised taxa. ${ }^{11}$ In addition, three isolates of $A$ equuli (one Larabinose positive and two L-arabinose negative) from horses and a pig outside Australia were included in the study. The type and reference strains for $A$ equuli, $P$ caballi and Bisgaard Taxon 9 were also included in this study.

\section{Materials and methods \\ Bacteria}

The isolates and the type and reference strains used are listed in Table 1. The 12 Australian isolates, which are held at the Animal Research Institute, have previously been characterised as either L-arabinose positive $A$ equuli, typical L-arabinose negative $A$ equuli, $P$ caballi or could not be assigned to any recognised taxa within the family Pasteurellaceae. ${ }^{11}$ The remaining three isolates were obtained from the culture collection held at the Royal Veterinary and Agricultural University in Denmark and have been shown to be either $A$ equuli or L-arabinose positive $A$ equuli (M Bisgaard unpublished). The type/reference strains for $A$ equuli, $P$ caballi and Bisgaard Taxon 9 were also obtained from the culture collection held at the Royal Veterinary and Agricultural University in Denmark.

\section{Bacterial growth and DNA extraction}

Isolates were grown overnight at $37^{\circ} \mathrm{C}$ in brain heart infusion broth (Difco). After this incubation, samples were cultured on blood agar (tryptose blood agar base, Difco, containing 5\% citrated bovine blood) to check for contamination. The bacteria were harvested by centrifugation. Chromosomal DNA was extracted from this pellet by a standard micro-method using cell lysis and phenol extraction as previously described. ${ }^{12}$

\section{Ribotyping}

Restriction digests were performed using the enzyme HaeIII. Ribotyping was performed using a probe based on the $16 \mathrm{~S}$ and $23 \mathrm{~S}$ rRNA of Escherichia coli as previously described. ${ }^{12}$

\section{Cluster analysis}

The ribotyping patterns were analysed by use of GelCompar (Applied Maths, Ghent, Belgium). The unweighted pair-group method clustering method of arithmetic averages clustering fusion strategy (UPGMA) and the Dice coefficient were used to create a phenogram to show the relationships between isolates. 
Table 1. Isolates and reference/type strains used in this study.

\begin{tabular}{|c|c|c|c|}
\hline Isolate & Source & Identification & Cluster \\
\hline $15445 / 4$ & Foal liver & $\mathrm{L}(+)$ arabinose ${ }^{+} A$ equuli & A \\
\hline J1959/4 & Horse foetal lung & $\mathrm{L}(+)$ arabinose ${ }^{+} A$ equuli & $A$ \\
\hline J786 & Foal liver & $\mathrm{L}(+)$ arabinose ${ }^{+} A$ equuli & $A$ \\
\hline S5632/4 & Horse kidney & $\mathrm{L}(+)$ arabinose ${ }^{+} A$ equuli & A \\
\hline Ac2 & Horse, Zimbabwe & $\mathrm{L}(+)$ arabinose ${ }^{+} A$ equuli & A \\
\hline NCTC8529' & Horse & A equuli & $\mathrm{B}$ \\
\hline Ac9a & Pig, Zimbabwe & A equuli & B \\
\hline $\mathrm{R} 4922 / 6$ & Horse liver & A equuli & $\mathrm{B}$ \\
\hline $\mathrm{R} 4922 / 7$ & Horse kidney & A equuli & $\mathrm{B}$ \\
\hline Ac10 & Horse, Zimbabwe & A equuli & $\mathrm{B}$ \\
\hline 93/107376/4 & Foal kidney & A equuli & B \\
\hline $14963 / 3$ & Horse joint & A equuli & B \\
\hline R3011/3 & Horse heart & A equuli & $\mathrm{B}$ \\
\hline F129 R & Horse oral cavity & Bisgaard Taxon 9 & C \\
\hline $\mathrm{H} 4272 / 4$ & Foal spleen & Unclassified & C \\
\hline $15054 / 5$ & Foal brain & Unclassified & D \\
\hline ATCC49197' & Horse uterus & P caballi & $E$ \\
\hline V2766 & Horse foetal stomach & P caballi & $E$ \\
\hline
\end{tabular}

Field isolates were obtained from Australian horses except isolates Ac2 and Ac10 which were obtained from horses in Africa and Ac9a from a pig in Africa.

$T$ = Type strain

$\mathrm{R}=$ Reference strain

\section{Results}

Clearly resolved patterns containing between 9 to 12 bands were obtained for all 15 field isolates and the three type and reference strains. A total of 17 different patterns could be detected by eye with R4922/6 and R4922/7 being the only isolates that shared a pattern.

Estimates of the degree of relation between the isolates are presented in the phenogram shown in Figure 1. Five clusters, termed A, B, C, D and E, are apparent at a similarity of approximately $50 \%$. All five isolates of $\mathrm{L}$-arabinose positive $A$ equuli were allocated to cluster $\mathrm{A}$. Within cluster $\mathrm{A}$, the non-Australian isolate of L-arabinose positive $A$ equuli (Ac2) was somewhat removed from the Australian isolates, joining the cluster at 57\% similarity. Cluster B consisted of all seven of the typical $A$ equuli isolates and the type strain of $A$ equuli, which is also $\mathrm{L}$-arabinose negative. In this group, the non-Australian equine isolate (Ac10) was not markedly different from the Australian isolates, falling in the middle of the cluster. The porcine isolate (Ac9a) was more closely related to the type strain of $A$ equuli than the equine isolates. Cluster $\mathrm{C}$ consisted of one of the two unclassified isolates plus the reference strain for Bisgaard Taxon 9. Cluster D consisted of the remaining unclassified isolate. Cluster E consisted of the sole P caballi isolate as well as the type strain for this species.

\section{Discussion}

Visual examination of the ribotyping patterns clearly showed considerable diversity among the isolates. Only isolates R4922/6 and R4922/7 showed a common pattern. As these two isolates were originally obtained from different tissues of the same horse, ${ }^{11}$ the finding of a common ribotype pattern was not unexpected. Indeed, the fact that these two isolates shared the same pattern indicates that the differences detected by ribotyping with the enzyme HaelII are most likely to be indicative of significant differences among isolates.

Computerised cluster analysis of the ribotyping patterns showed a marked diversity within the species $A$ equuli. Two

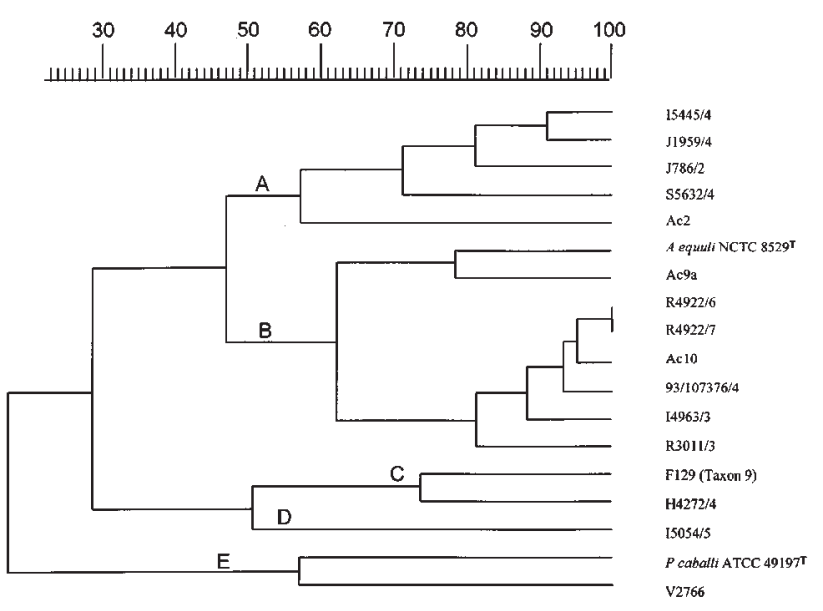

Figure 1. Phenogram of similarity amongst ribotyping patterns of $A$ equuli isolates clustered by UPGMA strategy. Numbering of isolates and strains corresponds to Table 1. Five clusters (A, B, C, D and E) are indicated at a similarity level of $55 \%$.

clusters were formed by the $A$ equuli isolates - one cluster (cluster A) consisted of only L-arabinose positive isolates while the second cluster (cluster B) consisted only of L-arabinose negative isolates plus the type strain for this species (which is also L-arabinose negative). The unique association of L-arabinose fermentation with cluster $\mathrm{A}$ indicates that production of acid from L-arabinose might be of taxonomic significance. Differences in $\mathrm{L}(+)$ arabinose fermentation also separate clusters C and D. ${ }^{11}$ Further work on a more extensive collection of field isolates from diverse sources are needed to determine the prevalence and significance of the ability to ferment L-arabinose in $A$ equuli.

The Australian isolates of $A$ equuli (whether L-arabinose positive or negative) were quite distinct from the two of the three overseas isolates and the type strain of the species. However, Ac10, a typical L-arabinose negative isolate obtained from a African horse, was found to cluster in with the typical L-arabinose negative field isolates from Australian horses. If the clusters outlined by ribotyping can be confirmed by other genetic methods, differences in $\mathrm{L}(+)$ arabinose, $\alpha$-galactosidase and trehalose can be used for separation of these taxa according to our previous study. ${ }^{11}$

The validity of the ribotype clusters is supported by the results obtained with the field isolate of $P$ caballi and the unclassified isolates. As reported previously, these three isolates were initially mistakenly identified as $A$ equuli. ${ }^{11}$ Only a careful reexamination with an extensive range of phenotypic tests was able to show the true nature of these isolates. ${ }^{11}$ The ribotype clustering was able to clearly show that these three isolates were quite distinct from the two types of $A$ equuli, with only a $30 \%$ similarity at best, confirming the results of our earlier phenotypic-based study. Of particular note is our finding that the sole field isolate of $P$ caballi (V2766) clustered with the type strain of this species. Overall, our results suggest that ribotyping could be very useful as a preliminary screening technique when examining $A$ equuli-like isolates from horses.

The results of this study have provided further evidence of the diversity within A equuli and similar organisms from horses. 
Our results suggest that the ability to ferment L-arabinose may be of some significance in $A$ equuli and related organisms. Further studies based on more definitive techniques such as DNA-DNA hybridisation and 165 rRNA sequencing are required to more clearly define this situation.

\section{References}

1. Bisgaard M. Ecology and significance of Pasteurellaceae in animals. Zentralbl Bakteriol 1993;279:7-26.

2. Golland LC, Hodgson DR, Hodgson JL et al. Peritonitis associated with Actinobacillus equuli in horses: 15 cases (1982-1992). J Am Vet Med Assoc 1994;205:340-343.

3. Radostits OM, Blood DC, Gay CC et al. Veterinary medicine. Baillière Tindall, London, 1993:1763.

4. Mutters R, Piechulla K, Hinz K-H, Mannheim W. Pasteurella avium (Hinz and Kunjara 1977) comb. nov. and Pasteurella volantium sp. nov. Int J Syst Bacteriol 1985;35:5-9.

5. Angen O, Caugant DA, Olsen JE, Bisgaard M. Genotypic relationships among strains classified with the Pasteurella haemolytica complex as indicated by ribotyping and multi-locus enzyme electrophoresis. Zentralbl Bakteriol 1997;286:333-354
6. Miflin JK, Horner RF, Blackall PJ et al. Phenotypic and molecular characterization of $\mathrm{V}$-factor (NAD)-independent Haemophilus paragallinarum. Avian Dis 1995;39:304-308.

7. Zhao G, Pijoan C, Murtaugh MP, Molitor TW. Use of restriction endonuclease analysis and ribotyping to study epidemiology of Pasteurella multocida in closed swine herds. Infect Immun 1992;60:1401-1405.

8. Fussing V, Wegener HC. Characterisation of bovine Haemophilus somnus by biotyping, plasmid profiling, REA-patterns and ribotyping. Zentralbl Bakteriol 1993;279:60-74.

9. Lester A, Gerner-Smidt P, Gahrn-Hansen B et al. Phenotypical characters and ribotyping of Pasteurella aerogenes from different sources. Zentralbl Bakteriol 1993;279:75-82.

10. Sedlácek I, Gerner-Smidt P, Schmidt J, Frederiksen W. Genetic relationship of strains of Haemophilus aphrophilus, $H$. paraphrophilus and Actinobacillus actinomycetemcomitans studied by ribotyping. Zentralbl Bakteriol 1993;279:5159.

11. Blackall PJ, Bisgaard M, McKenzie RA. Characterisation of Australian isolates of Actinobacillus capsulatus, Actinobacillus equuli, Pasteurella caballi and Bisgaard Taxa 9 and 11. Aust Vet $J$ 1997;75:52-55.

12. Christensen JP, Skov MN, Hinz K-H, Bisgaard M. Salmonella enterica serovar Gallinarum biovar gallinarum in layers: epidemiological investigations of a recent outbreak in Denmark. Avian Pathol 1994;23:489-501.

(Accepted for publication 13 May 1998)

\section{Verocytotoxigenic Escherichia coli in cats}

cute diarrhoea is a common sign in cats, but its pathogenesis is poorly understood. Apart from dietary, viral A and parasitic disorders, some bacteria are known to cause infectious gastroenteritis. They include salmonellae, Campylobacter sp, Bacillus piliformis and Clostridium perfringens. Recent studies have also implicated enteropathogenic $E$ coli.

Researchers from Guelph, Ontario, cultured faecal samples from 179 cats, 113 of which had diarrhoea, in a multicenter case-control study. Faecal cultures were tested for verotoxicity with a Vero cell assay and the type of verotoxin identified with PCR amplification. Verotoxin-positive isolates were serotyped and the prevalence of other bacterial and parasitic pathogens studied.

The overall prevalence of enteric verocytotoxigenic E coli (VTEC) was 12.3\%. Eleven of the VTEC-positive 22 cats had diarrhoea and 11 had normal stools. This suggests that VTEC are not a significant cause of diarrhoea in cats. The dominant verotoxin type was generic VT rather than VT1 and VT2, indicating that feline VTEC strains may have a verotoxin gene different to those producing VT1 and VT2. Eight VTEC serotypes were identified, four of which have been isolated in man. E coli 0157:H7, the dominant VTEC pathogenic for humans, was not among the isolates.

The samples yielded one case of salmonella and three of Campylobacter spp. The most common parasites were Toxocara cati (26 positive samples), Taenia (10) and Isospora (10).

VTEC, also known as shigatoxin-producing $E$ coli, have caused several devastating human outbreaks of bloody diarrhoea and haemolytic uraemic syndrome. VTEC are known to colonise healthy domestic animals and there is great potential for zoonotic transmission from contaminated foodstuffs. This study indicates that healthy and diarrhoeic cats may serve as a reservoir for human and bovine VTEC.

Smith KA, Kruth S, Hammermueller J, Gyles C, Wilson JB. A case-control study of verocytotoxigenic Escherichia coli infection in cats with diarrhoea. Can J Vet Res 1998;62:87-92. 